\title{
Improving localization precision of Brillouin measurements using spectral autocorrelation analysis
}

\author{
Eitan Edrei, Milos Nikolic and Giuliano Scarcelli* \\ Fischell Department of Bioengineering, University of Maryland \\ College Park, Maryland 20742, USA \\ *scarc@umd.edu
}

Received 30 June 2017

Accepted 21 August 2017

Published 18 September 2017

\begin{abstract}
Brillouin spectroscopy is a powerful tool for measuring the mechanical properties of materials without contact. The sensitivity to mechanical changes that a Brillouin spectrometer can detect is determined by the precision to which a spectral peak can be localized. The localization precision is however fundamentally limited by the low number of photons within a Brillouin measurement, as well as by intrinsic noise of the setup. Here, we present a method to improve the spectral sensitivity of Brillouin measurements by exploiting the autocorrelation function of the spectrum. We show that by performing a localization process on the autocorrelation function nearly $20 \%$ increase in localization precision can be obtained. This result is consistent between our theoretical treatment, numerical simulation and experimental results. We further study the effect of background noise on the precision improvement for realistic scenarios.
\end{abstract}

Keywords: Brillouin microscopy; localization.

\section{Introduction}

Brillouin light scattering has been used for many years to measure viscoelastic properties of materials without contact. ${ }^{1,2}$ Traditionally, Brillouin spectrometer utilizes a scanning interferometer featuring Fabry-perot etalons ${ }^{3-5}$; in recent years the introduction of a virtual image phased array (VIPA) etalons $^{6}$ and tilted geometry has dramatically enhanced the throughput of the spectrometer and allowed the acquisition of the entire Brillouin spectrum in one shot. ${ }^{7}$ The rapid acquisition of VIPAbased spectrometers enabled micro-spectroscopic mapping of the mechanical properties of samples by spatially scanning over an area of interest. ${ }^{8}$ Brillouin microscopy has found applications in the mechanical characterization of tissues ${ }^{9-12}$ and cells. ${ }^{13-17}$ Brillouin spectral analysis for applications that require high sensitivity to small mechanical changes is based on the localization of the spectral peak maximum. In fact, to capture biologicallyrelevant changes in material stiffness sensitivities of $\sim 10 \mathrm{MHz}$ are needed ${ }^{18}$; but both instrumentally, typical spectrometers have spectral resolutions in the order of hundreds of $\mathrm{MHz}$ to $\mathrm{GHz}$; and

*Corresponding author.

This is an Open Access article published by World Scientific Publishing Company. It is distributed under the terms of the Creative Commons Attribution 4.0 (CC-BY) License. Further distribution of this work is permitted, provided the original work is properly cited. 
fundamentally, biological materials present natural linewidths that exceed $500 \mathrm{MHz}$. Because of the localization processing, not only the spectral resolution of the spectrometer but also the signal-to-noise ratio and signal-to-background ratio are important factors that impact the spectral sensitivity of the measurement, which determines the mechanical sensitivity of the instrument. However, the intensity of the Brillouin spectral components is fundamentally limited due to the small scattering cross-section of the photon-phonon interaction. Over the past years, different approaches to this challenge have been pursued: on one hand stimulated scattering interaction ${ }^{19,20}$ or multiplexed configurations ${ }^{21}$ have been demonstrated; on the other hand, an ongoing effort is devoted to improving the efficiency of signal detection, ${ }^{22}$ spectral contrast of the spectrometer ${ }^{23-25}$ and removing background components. ${ }^{26-29}$ Here, we present an analysis method of the Brillouin spectrum based on the spectral autocorrelation function of the acquired signal. We will show both analytically and experimentally that working in the autocorrelation space is advantageous compared to current protocols in terms of localization precision.

\section{Principle}

Brillouin spectra are usually shown as a triplet of peaks: the central nonshifted peak arises from elastic scattering, while two shifted peaks, named Stokes and anti-Stokes, come from the inelastic phonon-photon scattering interactions. To determine the local elasticity from the acquired spectrum the shift of the Stokes or anti-Stokes peaks from the nonshifted central peak is required. However, the central peak is often many orders of magnitude brighter than the Brillouin signals as it contains Rayleigh, Mie interactions as well as all backreflections of laser within the setup; therefore, the dynamic range of the camera cannot capture both laser and Brillouin peaks in the same frame. One way to address this problem is to physically block the central peak in order to measure the weaker Brillouin peak. Under these circumstances though, it is impossible to determine the shift of the Brillouin signal, as no reference from the laser is recorded. A potential solution to this issue is to record the location of the laser peak beforehand, and use it as a reference to evaluate the Brillouin shift in future measurements. However, laser frequency drifts or spectrometer jitters during measurements are difficult to avoid so that the laser peak location cannot be assumed to be constant. To resolve this issue, one can take advantage of the fact that Brillouin spectrometers show several diffraction orders, therefore, the Stokes and anti-Stokes peaks from two different diffraction orders can be captured in a single acquisition: the distance between the two peaks represents a robust signature to determine the Brillouin shift.

The straightforward procedure to obtain the Brillouin shift in these conditions is as follows: the spectrum containing the Brillouin Stokes and anti-Stokes peaks from two consecutive diffraction orders is recorded, and the distance between the peaks is determined by localizing each peak using a fitting algorithm. Next, the peak separation is subtracted from the known free spectral range of the spectrometer, and this value corresponds to twice the magnitude of the Brillouin shift in pixels. To transform the result into frequency units, a calibration process is carried-out using materials of known Brillouin shifts. ${ }^{13,22}$ Although the Brillouin peaks natural bandwidth is typically hundreds of $\mathrm{MHz}$, the precision to which the center of the peaks can be determined is much greater. Similar to localization microscopy and particle tracking, the localization accuracy of the center of a spot is determined by optical properties such as the number of photons within the spot and the background noise present in the measurement. ${ }^{30}$ In contrast to localization microscopy protocols, however, in which the absolute location of each emitter is desired, the key parameter in Brillouin analysis is the relative distance between the Stokes and antiStokes peaks from which the Brillouin shift can be extracted. Although the peak separation can be obtained by performing two separate localization processes as described above, each localization process will contribute to the error of the estimation of the peaks separation which will therefore be enhanced. Here, we demonstrate a different approach which relies on the autocorrelation function of the acquired spectrum. The autocorrelation of an object preserves information about the relative distances within the object, while the actual object form is lost and can be retrieved only under specific circumstances. ${ }^{31-34}$ Particularly, for a two-peak spectrum the autocorrelation will contain three peaks, and the distance between the original two peaks 


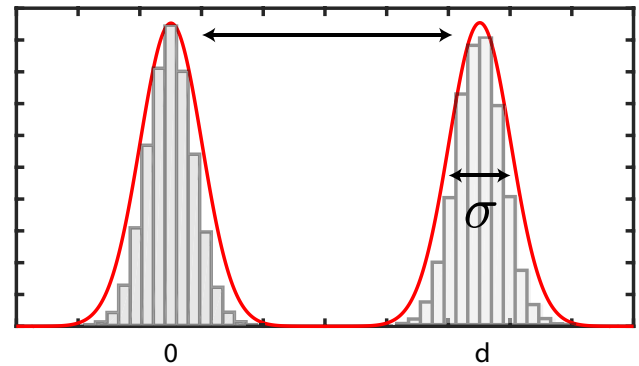

(a)

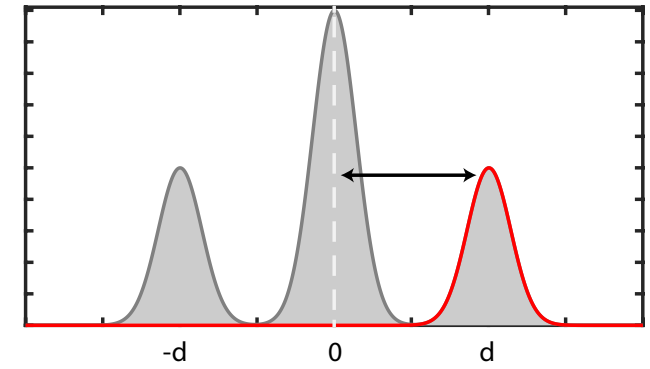

(b)

Fig. 1. (a) Original signal: the distance between two peaks " $d$ " can be obtained by fitting the data and measuring the separation. (b) The autocorrelation function: the displacement of either side peaks from the center equals the distance between the original peaks.

will transform into the distance between the center of the autocorrelation function and either side peak Figs. 1(a) and 1(b). Hence, tracking the distance between the two spectral peaks is equivalent to tracking the distance of the side peak from the center in the autocorrelation space. This results in improved precision in the localization protocol, thus offering a more efficient use of the information content of the collected photons.

\section{Theory}

In general terms, the spatial uncertainty on the detection of a single photon hitting a camera is determined by the standard deviation of the point spread function of the imaging system $\sigma$. Yet, a set of $N$ independent measured photons can be treated as $N$ measurements of the signal location, hence, the precision to which the average position can be determined is given by the standard error of the mean: $\frac{\sigma}{\sqrt{N}}$. When two signals are detected and the distance between the signals is of interest, it can be measured using two separate localization processes or, as proposed here, by a single localization process in the autocorrelation space. Although the Brillouin signal presents a natural Lorentzian spectral distribution, the signal is convolved with the Airy pattern generated by the imaging systems of the spectrometer, as well as with a Gaussian envelope attributed to the apodization of the VIPA output. Therefore, a treatment of a more complex Voight profile will be the most proper one, yet, for simplicity here we evaluate the localization precision for both the double fitting and the autocorrelation analysis and compare them for one-dimensional Gaussian shaped signals.
We begin by calculating the precision to which the distance between two signals can be determined. We consider a function of two independent Gaussian shaped signals separated by a distance " $d$ " as presented in Fig. 1(a):

$$
f(x)=\frac{N_{o}}{\sigma \sqrt{2 \pi}}\left(e^{\frac{-(x-d) 2}{2 \sigma^{2}}}+e^{\frac{-x^{2}}{2 \sigma^{2}}}\right),
$$

where $N_{o}$ is the number of photons contained in each peak. Hence, the localization precision of each peak is given by the standard error of the mean for the calculated number of photons: $\frac{\sigma}{\sqrt{N_{o}}}$. The peak separation error is influenced by both peak localizations and is therefore larger:

$$
\sqrt{\left\langle(\Delta d)^{2}\right\rangle} \text { Double fit }=\frac{\sqrt{2} \sigma}{\sqrt{N}_{o}} .
$$

Next, we derive the localization accuracy for the autocorrelated function. The autocorrelation can be calculated by multiplying Eq. (1) by a shifted form of itself and integrating over the spatial coordinate:

$$
\begin{aligned}
C(q)= & \int_{-\infty}^{\infty} \frac{N_{o}}{\sigma \sqrt{2 \pi}}\left(e^{\frac{-(x-d)^{2}}{2 \sigma^{2}}}+e^{\frac{-x^{2}}{2 \sigma^{2}}}\right) \\
& \cdot \frac{N_{o}}{\sigma \sqrt{2 \pi}}\left(e^{\frac{-(x-d-q) 2}{2 \sigma^{2}}}+e^{\frac{-(x-q) 2}{2 \sigma^{2}}}\right) d x,
\end{aligned}
$$

where $q$ is the spatial coordinate in the autocorrelation space and represents the displacement of the two functions. The autocorrelation, normalized to the single peak intensity, can be therefore written as:

$$
C(q)=\frac{N_{o}}{\sigma \sqrt{2 \pi}}\left(e^{\frac{-(d+q) 2}{4 \sigma^{2}}}+2 e^{\frac{-q^{2}}{4 \sigma^{2}}}+e^{\frac{-(d-q) 2}{4 \sigma^{2}}}\right) .
$$

The autocorrelation function has three peaks, each side peak is located a distance " $d$ " from the center 
of the function corresponding to the original separation of the peaks (Fig. 1(b)). To evaluate the localization precision for the correlated peak, we calculate the effective number of photons in the side peak of the autocorrelation by integrating over one of the side peaks:

$$
\int \frac{N_{o}}{\sigma \sqrt{2 \pi}} e^{\frac{-(d-q) 2}{4 \sigma^{2}}} d q=\sqrt{2} N_{0} .
$$

The localization precision is determined by the standard error of the mean in the new space; it thus depends on the number of photons calculated in (5) and the width of the autocorrelation peak, which is $\sqrt{2} \sigma$. As a result, the localization precision is therefore:

$$
\sqrt{\left\langle(\Delta d)^{2}\right\rangle} \text { Autocorrelation }=\frac{\sqrt{2} \sigma}{\sqrt{\sqrt{2} N_{0}}} .
$$

The net improvement in precision provided by the autocorrelation process is therefore:

$$
\frac{\sqrt{\left\langle(\Delta d)^{2}\right\rangle}}{\left.\sqrt{\left\langle(\Delta d)^{2}\right\rangle}\right\rangle_{\text {Autocorrelation }}}=\sqrt{\sqrt{2}} .
$$

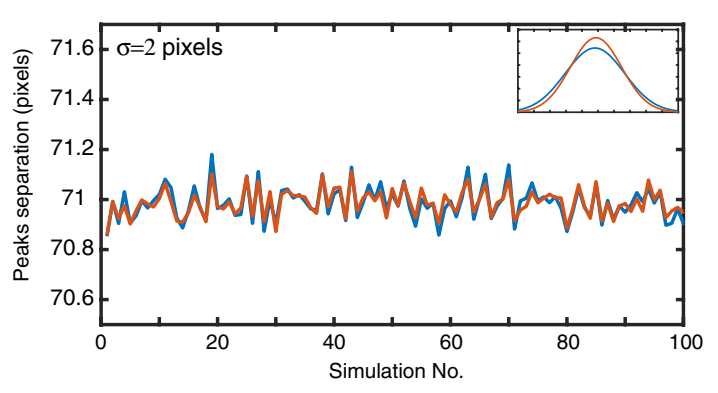

(a)

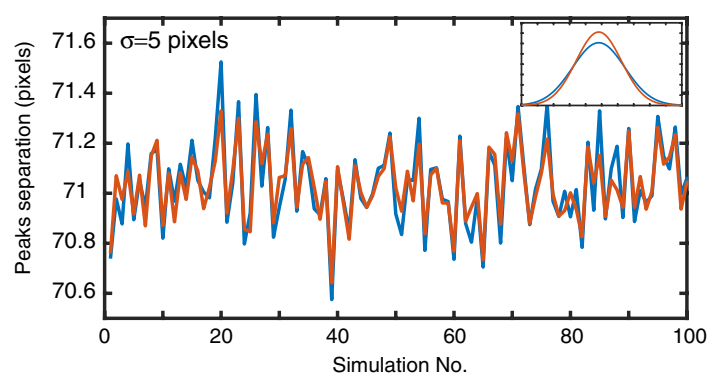

(b)
This can be understood intuitively: the localization improvement is a result of the doubled number of photons used to localize the correlated peak; the signal processing of each original peak involves half the number of photon used by the correlation process in which the photons from both individual signals are considered. However, the localization precision is not improved by a factor of $\sqrt{2}$ as expected when the number of photons is doubled, because the multiplication process translates into a precision loss. Note that by performing a correlation process on an acquired data with two independent signals, the information about the locations of the peaks is lost, yet, the distance between the peaks can be easily extracted which is sufficient in our scenario.

To support our derivation, we performed a numerical simulation of the localization process. We generated many Brillouin spectra with two Gaussian shaped signals (resembling the Stokes and anti-Stokes peaks), and assigned a Poisson noise behavior to each signal. We determined the distance between the signals both by localizing them using a fit for two Gaussians and by performing an

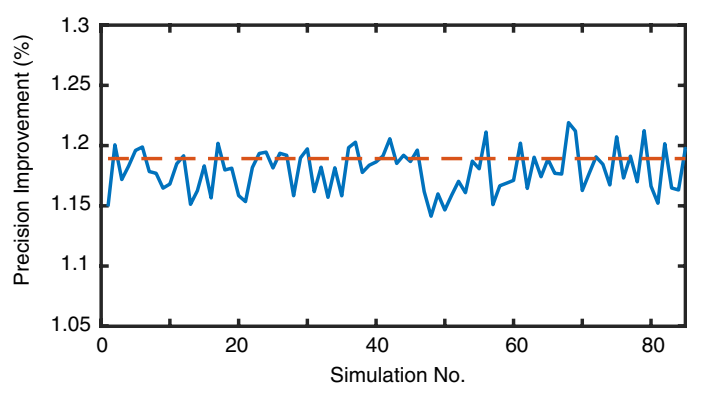

(c)

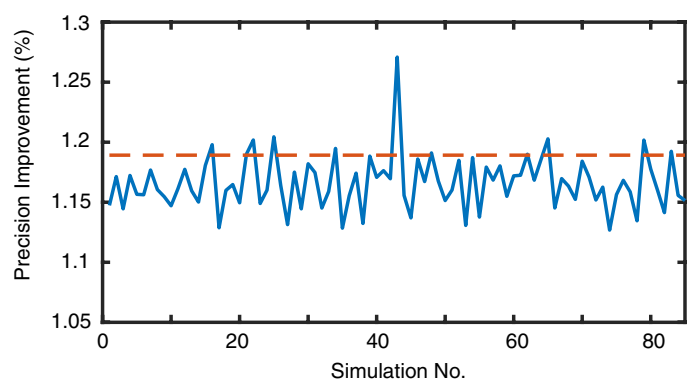

(d)

Fig. 2. (Color online) Simulation results: (a) Two peaks two pixels wide were repeatedly generated, and the distance between them was measured using a double peak fitting (blue curve) and a single fit of the autocorrelation function (red curve). The inset shows the corresponding histogram for the two measurements. (b) A similar measurement as in (a), with five pixels wide peaks. (c) The standard deviation of the data presented in (a) was measured repeatedly and the ratio between the standard deviations of the two analyses was quantified (blue curve). The theoretical expected improvement (red dashed line) is in good agreement with the simulated results. (d) A similar measurement as in (c), with five pixels wide peaks. 
autocorrelation and localizing a single side peak. Figures 2(a) and 2(b) show the result for signals of widths 2 and 5 pixels, respectively. It can be seen that the red curve corresponding to the autocorrelation fit has a smaller standard deviation than the blue curve which was obtained using a double peak fitting. To better observe the differences, we plotted a histogram of both curves, as expected, the red curve transforms into a sharper and narrower histogram (Figs. 2(a) and 2(b) insets). To compare our simulation results to the expected theoretical ones, we measured the standard deviation for many sets of measurements, and calculated the precision improvement which is the ratio of the standard deviations of the fitting results. We used a signal width of 2 (Fig. 2(c)) and 5 (Fig. 2(d)) pixels for this simulation and found an average accuracy improvement of $\sim 18 \%$ and $\sim 17 \%$, respectively, in good agreement with the theoretical prediction (Figs. 2(c) and 2(d), red dashed line).

Here, we derived and studied numerically the localization precision of two identical signals, since, due to error propagation considerations, it is preferable to set the system to yield identical signals rather than having an uneven photon distribution.

\section{Experimental Data}

To verify experimentally our results, we built the setup illustrated in Fig. 3(a). We expanded a single frequency laser beam of wavelength $532 \mathrm{~nm}$ (LaserQuantum), and transmitted it through a polarizing beam splitter (PBS) and a quarter waveplate. We focused the beam onto a sample using a one inch lens $(f=50 \mathrm{~mm})$. The back reflected light was collected in an epi-detection configuration, and after a second transmission through the quarter wave-plate, it acquired a polarization state of $90^{\circ}$ with respect to the incoming beam. We used the PBS to direct the reflected light into the back entrance of an objective lens $(20 \times, 0.4 \times \mathrm{NA})$ and coupled it into a single mode fiber. The output of the fiber was spectrally analyzed using a double stage VIPA spectrometer with a $30 \mathrm{GHz}$ free-spectral-range (for details see Ref. 23).

Figure 3(b) shows a representative spectrum acquired using methanol as a sample (blue dots), and a matching double peak fit (red curve). The autocorrelation of the spectrum acquired in Fig. 3(b) is presented in Fig. 3(c) (blue dots), as well as a matching single fit (red curve). To quantify the localization precision in both cases, we repeated the (a)

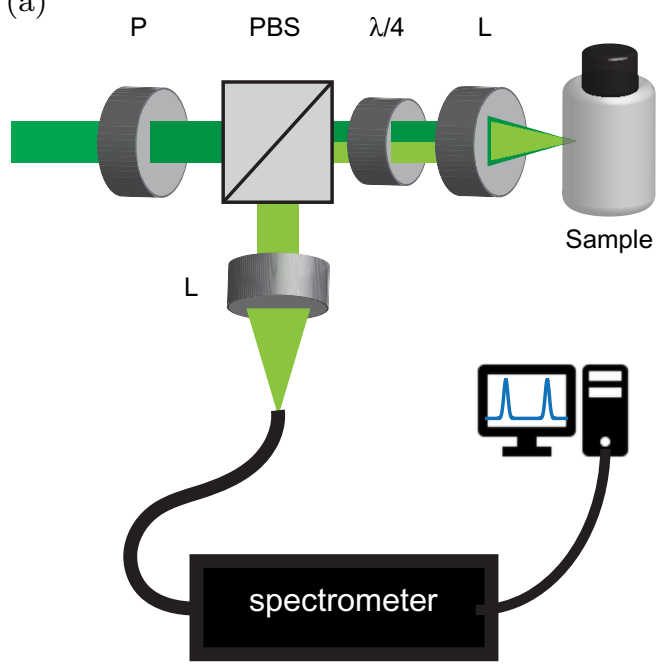

(b)

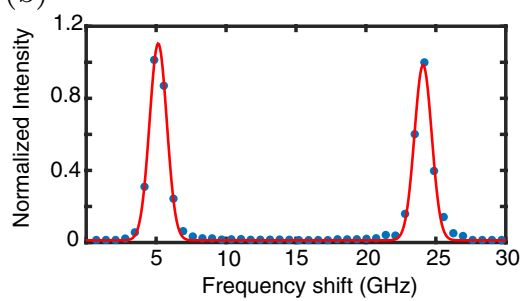

(d)

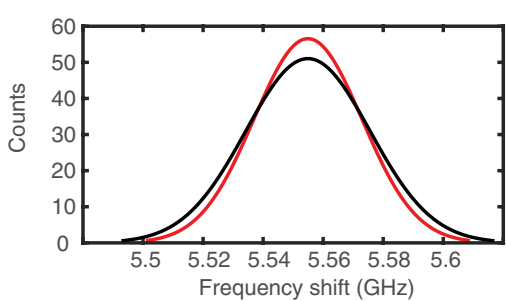

(c)

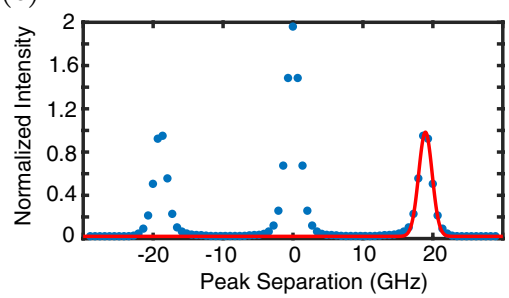

(e)

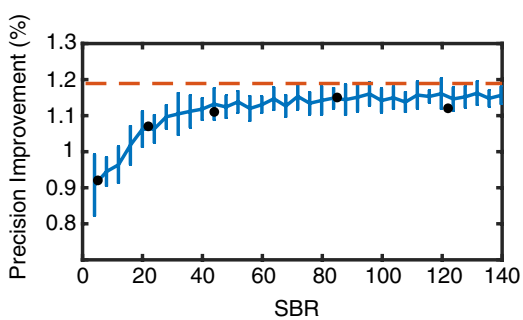

Fig. 3. (Color online) (a) Experimental setup: an epi-detection configuration was used to illuminate the sample (dark green path) and collect the back scattered light (bright green path). The collected light was spectrally analyzed using a double stage VIPA spectrometer. (b) A typical acquired spectrum of a methanol sample — blue dots (laser intensity 10 mW, $40 \mathrm{~ms}$ integration time), and a double peak Gaussian fit — red curve. (c) The autocorrelation function of the spectrum shown in (b) — blue dots, and a single peak fit of the side peak - red curve. (d) A histogram of the separation between the peaks shown in (b) for 500 measurements (black curve), and a matching histogram of the distance of the side peak in (c) from the center of the autocorrelation function (red curve). (e) Background effect on the precision improvement: at low SBR values the autocorrelated process is less accurate than the double fitting one, while at high SBR values the precision improvement (blue curve) approaches the theoretical one (red dashed line). The experimental data (black dots) agree with the numerical simulation (blue curve). 
measurement 500 times and presented the histogram of the fluctuations in Fig. 3(d). As predicted previously, the precision of the single autocorrelated peak is higher resulting in a narrower histogram (Fig. 3(d), red curve) compared with the double fitting of the acquired spectrum (Fig. 3(d), black curve).

In the analytical derivation and the numerical simulation, it was assumed that every pixel of the signal is shot-noise limited. However, in practice, background noise is inevitable. To examine the effect of background noise on our results, we repeated the numerical study with varying levels of signal to background ratios (SBR). To do so, we varied the total number of photons in every peak within the simulation from 100 to 3500 , a range similar to the experimental one, and used a constant background of one photon which is a typical experimental value with high-end CCD or sCMOS cameras. Figure 3(e) shows the localization precision improvement due to the autocorrelation process as a function of the SBR (blue curve), and theoretical shot-noise limited improvement (red dashed line). We found that for high SBR values the improvement approaches the theoretical prediction, while for $\mathrm{SBR}<20$, the correlation process becomes less accurate than the double fitting procedure. To confirm this prediction, we repeated the experimental study with different SBR values by adjusting the integration time of the camera, and found a good agreement with the simulated plot (Fig. 3(e), black dots). In realistic experimental situations, the localization of the autocorrelation peak yielded $\approx 15 \%$ higher precision than the double fit analysis.

\section{Discussion}

The precision to which an optical signal can be spatially localized has been studied for many years, ${ }^{30}$ and is the underlying principle for superresolution localization microscopy techniques such as PALM ${ }^{35}$ and STORM. ${ }^{36}$ Brillouin spectroscopy also uses this type of localization process thus achieving spectral sensitivities that are much higher than the nominal spectral resolution of the spectrometer. However, Brillouin spectroscopy presents a peculiar situation where the relevant quantity is the distance between two acquired signals rather than the absolute location of a single peak. In these conditions, autocorrelation functions are particularly suitable for signal analysis. By transforming to the autocorrelation space, the spatial form of the original function is lost, however, the information regarding the distances between locations is preserved. Here, we have shown that the localization process yields higher precision when applied to the autocorrelation function instead of the original signals.

We have compared the current method of spectral analysis to the autocorrelation both theoretically, numerically and experimentally. The analytical derivation predicts an improved precision of $\sim 18.9 \%$ for the autocorrelated analysis, which is in good agreement with our experimental data. For instance, for the data presented in Figs. 3(b)-3(d), we measured the width of the original signal to be $\sim 850 \mathrm{MHz}$, while the localization precision obtained by the double fitting process was $\sim 41.1 \mathrm{MHz}$ and the precision of the fit of the autocorrelated data gave an advantage of $\sim 15 \%$, i.e., $35.7 \mathrm{MHz}$. We also found that, the background imposes a limit on the expected improvement obtained by the autocorrelation processing. Indeed, we found that at low SBRs autocorrelation ceases to provide an advantage. This effect is attributed to the fact that through the autocorrelation process background photons from all locations within the spectrum contribute to the signal localization. Hence, using the autocorrelation analysis will be advantageous only when applied to high SBR scenarios, while for low SBR measurements such as within scattering medium, the double fitting approach will yield a higher localization precision.

Previous studies on localization precision have shown the dependency of the final sensitivity on acquisition parameters such as the number of detected signal and background photons as well the ratio between the camera pixel size and the point spread function of the signal. ${ }^{30,37}$ In terms of signal photons, the Brillouin spectroscopy application poses strong constraints in the spontaneous regime; in this paper, we have used realistic parameters of $\sim 3000$ total number of photons per peak which is about what is obtained in typical samples with a few $\mathrm{mW}$ incident light and $100 \mathrm{~ms}$ of integration time; as of background photons, these limits are imposed by the camera utilized and the experimental setup, we used an EMCCD camera (Andor iXon 897) with an intrinsic background noise $<1$ photon, hence, in our simulation we used an upper limit background of one photon. As for the ratio 


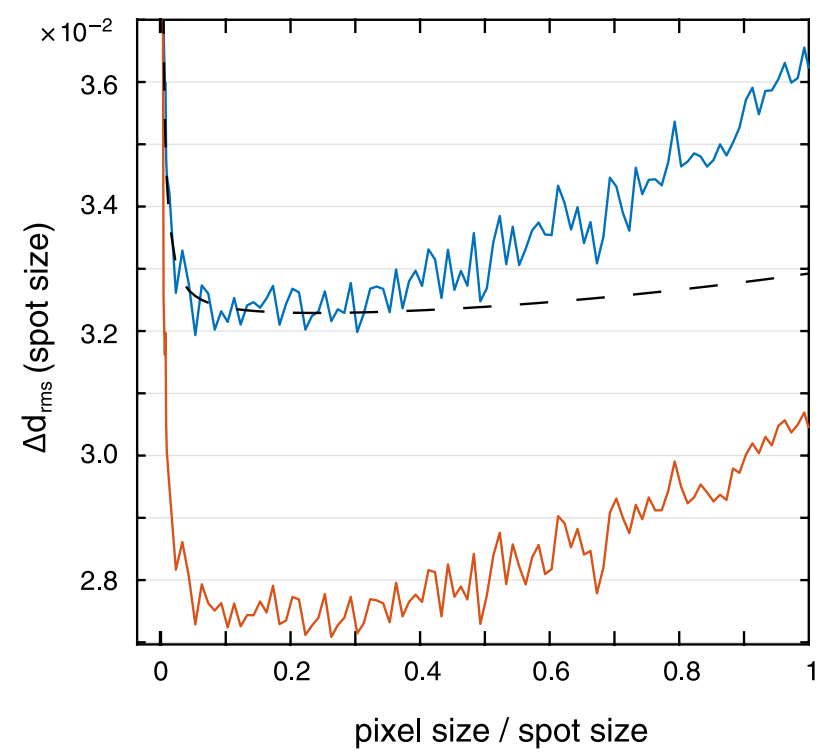

Fig. 4. (Color online) Monte Carlo simulation of peak distance precision (percentage of the spot size) as a function of the ratio between the pixel size and the spot size for the double fitting analysis (blue) and the autocorrelation analysis (red). The simulated results present similar behavior to the theoretical expectation (black dashed line) for small pixels sizes.

between the camera pixel size and the point spread function of the signal, it has been shown that the optimal choice depends on the specific parameters as well as the dimensionality of the measurement. ${ }^{30}$ In order to evaluate the effect of the autocorrelation on this behavior, we used a Monte Carlo simulation to generate signals with different ratios of pixels-toPSF widths using the above-mentioned parameters. The results are presented in Fig. 4. Both the autocorrelation analysis (red) and the double fitting approach (blue) yielded a similar optimal ratio of $\sim 4$ pixels per PSF while preserving the $\sim 20 \%$ precision improvement of the autocorrelation process. We also compared the simulated results to the analytical expectation derived in Ref. 30, as presented in the black dashed line in Fig. 4. It can be seen that the analytical expectation is in good agreement with the simulated results for small pixels, as the pixels become larger there is an underestimation of the localization error which is a result of a first-order approximation approach of the analytical derivation (see Ref. 30).

It should be noted that Fig. 4 is a proper estimation of the localization precision for a onedimensional spectral scenario, for a two-dimensional case, the optimal point is obtained at $\sim 2$ pixels per
PSF. Another consideration for Brillouin spectral localization is that different materials have different linewidths, therefore a compromise needs to be reached by choosing spectrometer alignment that fits best the material under examination.

\section{References}

1. J. G. Dil, "Brillouin scattering in condensed matter," Rep. Progr. Phys. 45(3), 285-334 (1982).

2. J. R. Sandercock, "Light-scattering from surface acoustic phonons in metals and semiconductors," Solid State Commun. 26(8), 547-551 (1978).

3. J. R. Sandercock, "Some recent developments in Brillouin scattering," Rca Rev. 36(1), 89-107 (1975).

4. J. M. Vaughan, J. T. Randall, "Brillouin scattering, density and elastic properties of the lens and cornea of the eye," Nature 284(5755), 489-491 (1980).

5. K. J. Koski, P. Akhenblit, K. McKiernan, J. L. Yarger, "Non-invasive determination of the complete elastic moduli of spider silks," Nat. Mater. 12 (3), 262-267 (2013).

6. M. Shirasaki, "Large angular dispersion by a virtually imaged phased array and its application to a wavelength demultiplexer," Opt. Lett. 21(5), 366368 (1996).

7. G. Scarcelli, S. Yun, "Multistage VIPA etalons for high-extinction parallel Brillouin spectroscopy," Opt. Express 19(11), 10913-10922 (2011).

8. G. Scarcelli, S. H. Yun, "Confocal Brillouin microscopy for three-dimensional mechanical imaging," Nat. Photon. 2(1), 39-43 (2008).

9. G. Scarcelli, P. Kim, S. Yun, "In vivo measurement of age-related stiffening in the crystalline lens by Brillouin optical microscopy," Biophys. J. 101(6), 1539-1545 (2011).

10. G. Scarcelli, R. Pineda, S. Yun, "Brillouin optical microscopy for corneal biomechanics," Invest. Ophthalmol. Vis. Sci. 53(1), 185-190 (2012).

11. F. Palombo et al., "Chemico-mechanical imaging of Barrett's oesophagus," J. Biophoton. 9(7), 694-700 (2016).

12. G. Antonacci et al., "Quantification of plaque stiffness by Brillouin microscopy in experimental thin cap fibroatheroma," J. R. Soc. Interface 12(112), 20150843 (2015).

13. G. Scarcelli et al., "Noncontact three-dimensional mapping of intracellular hydromechanical properties by Brillouin microscopy," Nat. Methods 12(12), 1132-1134 (2015).

14. J. Zhang, X. Nou, H. Kim, G. Scarcelli, "Brillouin flow cytometry for label-free mechanical phenotyping of the nucleus," Lab Chip. 17(4), 663-670 (2017). 
15. G. Antonacci, S. Braakman, "Biomechanics of subcellular structures by non-invasive Brillouin microscopy," Sci. Rep. 6, 37217 (2016).

16. Z. Meng, S. Lopez, K. Meissner, V. Yakovlev, "Subcellular measurements of mechanical and chemical properties using dual Raman-Brillouin microspectroscopy," J. Biophoton. 9(3), 201-207 (2016).

17. K. Elsayad et al., "Mapping the subcellular mechanical properties of live cells in tissues with fluorescence emission-Brillouin imaging," Sci. Signal. 9(435), (2016), Art. no. ARTN rs5.

18. G. Scarcelli, S. Besner, R. Pineda, P. Kalout, S. H. Yun, "In vivo biomechanical mapping of normal and keratoconus corneas," JAMA Ophthalmol. 133, 480-482 (2015).

19. C. Ballmann, J. Thompson, A. Traverso, Z. Meng, M. Scully, V. Yakovlev, "Stimulated Brillouin scattering microscopic imaging," Sci. Rep. 5, 18139 (2015).

20. I. Remer, A. Bilenca, "High-speed stimulated Brillouin scattering spectroscopy at $780 \mathrm{~nm}$," Appl. Photon. 1(6), 061301 (2016).

21. J. Zhang, A. Fiore, S. Yun, H. Kim, G. Scarcelli, "Line-scanning Brillouin microscopy for rapid noninvasive mechanical imaging," Sci. Rep. 6, 35398 (2016).

22. K. V. Berghaus, S. H. Yun, G. Scarcelli, "High speed sub-GHz spectrometer for Brillouin scattering Analysis," Jove-J. Vis. Exp. 106, 53468 (2015).

23. E. Edrei, M. Gather, G. Scarcelli, "Integration of spectral coronagraphy within VIPA-based spectrometers for high extinction Brillouin imaging," Opt. Express 25, 6895 (2017).

24. A. Fiore, J. Zhang, P. Shao, S. Yun, G. Scarcelli, "High-extinction virtually imaged phased arraybased Brillouin spectroscopy of turbid biological media," Appl. Phys. Lett. 108(20), 203701 (2016).

25. P. Shao, S. Besner, J. Zhang, G. Scarcelli, S. Yun, "Etalon filters for Brillouin microscopy of highly scattering tissues," Opt. Express 24(19), 22232-22238 (2016).

26. K. Berghaus, J. Zhang, S. H. Yun, G. Scarcelli, "High-finesse sub-GHz-resolution spectrometer employing VIPA etalons of different dispersion," Opt. Lett. 40(19), 4436-4439 (2015).

27. I. Remer, A. Bilenca, "Background-free Brillouin spectroscopy in scattering media at $780 \mathrm{~nm}$ via stimulated Brillouin scattering," Opt. Lett. 41(5), 926-929 (2016).

28. G. Antonacci, G. Lepert, C. Paterson, P. Torok, "Elastic suppression in Brillouin imaging by destructive interference," Appl. Phys. Lett. 107(6), 061102 (2015).

29. Z. Meng, A. Traverso, V. Yakovlev, "Background clean-up in Brillouin microspectroscopy of scattering medium," Opt. Express 22(5), 5410-5415 (2014).

30. R. E. Thompson, D. R. Larson, W. W. Webb, "Precise nanometer localization analysis for individual fluorescent probes," Biophys. J. 82(5), 27752783 (2002).

31. J. Fienup, "Reconstruction of an object from modulus of its Fourier-transform," Opt. Lett. 3(1), 27-29 (1978).

32. O. Katz, P. Heidmann, M. Fink, S. Gigan, "Noninvasive single-shot imaging through scattering layers and around corners via speckle correlations," Nat. Photon. 8(10), 784-790 (2014).

33. E. Edrei, G. Scarcelli, "Optical imaging through dynamic turbid media using the Fourier-domain shower-curtain effect," Optica 3(1), 71-74 (2016).

34. J. Bertolotti, E. van Putten, C. Blum, A. Lagendijk, W. Vos, A. Mosk, "Non-invasive imaging through opaque scattering layers," Nature 491(7423), 232234 (2012).

35. E. Betzig et al., "Imaging intracellular fluorescent proteins at nanometer resolution," Science $\mathbf{3 1 3}$ (5793), 1642-1645 (2006).

36. M. J. Rust, M. Bates, X. Zhuang, "Sub-diffractionlimit imaging by stochastic optical reconstruction microscopy (STORM)," Nat. Methods 3(10), 793795 (2006).

37. R. J. Ober, S. Ram, E. S. Ward, "Localization accuracy in single-molecule microscopy," Biophys. J. 86(2), 1185-1200 (2004). 